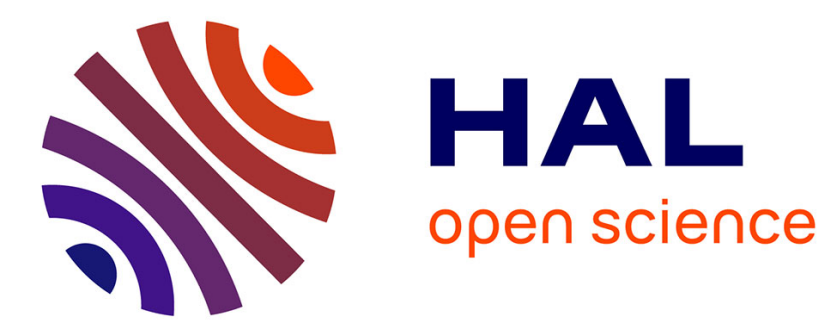

\title{
Le printemps des partis? Le rôle des organisations partisanes égyptiennes dans les élections législatives
}

\author{
Clément Steuer
}

\section{To cite this version:}

Clément Steuer. Le printemps des partis? Le rôle des organisations partisanes égyptiennes dans les élections législatives. Confluences Méditerranée , 2012, 82, pp.91-105. halshs-00839689

\section{HAL Id: halshs-00839689 \\ https://shs.hal.science/halshs-00839689}

Submitted on 28 Jun 2013

HAL is a multi-disciplinary open access archive for the deposit and dissemination of scientific research documents, whether they are published or not. The documents may come from teaching and research institutions in France or abroad, or from public or private research centers.
L'archive ouverte pluridisciplinaire HAL, est destinée au dépôt et à la diffusion de documents scientifiques de niveau recherche, publiés ou non, émanant des établissements d'enseignement et de recherche français ou étrangers, des laboratoires publics ou privés. 


\title{
Le printemps des partis ? Le rôle des organisations partisanes égyptiennes dans les élections législatives
}

\author{
Clément Steuer \\ Chercheur associé au Cedej et au laboratoire Triangle, Clément Steuer est soutenu par une bourse de \\ la Région Rhône-Alpes
}

Les partis politiques égyptiens ont joué un rôle clef durant la phase de transition. En effet, et bien qu'ils aient été largement discrédités dans les années qui ont précédé la révolution, ils ont fait l'objet d'un véritable engouement dans les mois qui ont suivi le départ de Moubarak. Du fait du nouveau contexte juridique issu de cette révolution ils ont en outre été amenés à jouer un rôle inédit au cours des élections législatives de 2011-2012, notamment en matière de sélection des candidats et de mobilisation des électeurs. Malgré tout, les partis politiques égyptiens n'ont pas été les seuls acteurs de ces élections, et ont dû composer avec d'autres formes d'organisation, porteuses de logiques différentes, ce qui les a conduits à adapter leurs stratégies afin de tirer le meilleur parti possible des dites logiques.

Cet article repose sur des observations faites essentiellement au Caire au printemps et à l'automne 2011 - directement auprès des partis politiques, mais également de syndicats et d'associations, ainsi que dans différentes manifestations -, et sur trois enquêtes de terrain d'une durée de deux semaines chacune, menées dans trois circonscriptions électorales au cours des élections à l'assemblée du peuple de 2011-2012 : Qasr Al-Nîl (centre-ville du Caire), Suez, et la première circonscription de Gharbiya, dans le Delta du Nil (Tanta et ses environs).

\section{Le printemps post-révolutionnaire des organisations partisanes}

La floraison de nouveaux partis est un phénomène somme toute banal dans les périodes de transition démocratique ${ }^{1}$. Il est cependant particulièrement remarquable dans l'Égypte de 2011 pour deux raisons : le discrédit qui frappait la forme partisane avant la révolution ${ }^{2}$, et l'hostilité traditionnelle de certains acteurs politiques parmi les plus importants à l'égard de ce mode d'organisation. Le nouveau contexte politique et juridique né de la révolution a néanmoins suscité une effervescence autour de la création de nouveaux partis.

\section{Le discrédit frappant la forme partisane à la veille de la révolution}

Les symptômes et les causes de la faiblesse des partis politiques égyptiens sont bien documentées depuis désormais une quinzaine d'années ${ }^{3}$ : déficit organisationnel et manque de moyens humains et financiers étaient causés par les règles autoritaires encadrant leurs activités et par le caractère clientéliste des transactions électorales. Ainsi, la création d'un parti devait obtenir l'approbation de la commission des partis, dont la majorité des membres étaient nommée directement ou indirectement par le président de la République. Cette commission pouvait également à tout moment suspendre l'activité d'un parti existant, en vertu de la loi $\mathrm{n}^{\circ} 40$ de 1977 sur les partis politiques, dont le flou des dispositions garantissait dans les faits un pouvoir quasi-discrétionnaire aux dirigeants du régime sur la vie partisane. Par ailleurs, l'application de l'état d'urgence - sans

1 Voir par exemple le dossier « Partis politiques et changement de régime », coordonné par Myriam Aït-Aoudia et Jérôme Heurtaux, dans Critique internationale, $\mathrm{n}^{\circ}$ 30, 2006.

2 Sur ce point, voir Steuer Clément, «L'apparent épuisement de la forme partisane », in Lavergne Marc (dir.), Chroniques égyptiennes 2009-2010, 2012, pp. 189-218.

3 Ben Nefissa Sarah, « Les Partis politiques égyptiens entre les contraintes du système politique et le renouvellement des élites », Revue des Mondes Musulmans et de la Méditerranée, 1998, pp. 55-87. Et plus récemment, Abdul Rahman Amr, "The Opposition Parties Crisis or the Crisis of Liberal Democracy", in Klaus Enrique et Chaymaa Hassabo (dir.), Chroniques égyptiennes 2006, Éditions du Cedej, Le Caire, 2007, pp. 143-174. 
cesse renouvelé depuis 1981 - permettait à l'exécutif d'interdire aux partis politiques d'organiser toute manifestation publique.

Ainsi, à la veille de la révolution, il n'existait en Égypte que 24 partis d'opposition légaux, dont la plupart n'avaient pas d'existence réelle hormis leur reconnaissance par le régime, qui pouvait parfois se traduire par quelques sièges dans l'une ou l'autre des deux assemblées élues (assemblée du peuple et assemblée consultative). En effet, la puissance économique et sociale des notables ralliés au parti national démocratique (PND), l'ampleur des fraudes et - à partir de 2000 - le recours de plus en plus répandu à la violence électorale, assuraient au pouvoir une maîtrise presque totale sur la composition des deux chambres du Parlement. Dans les faits, la véritable concurrence se jouait davantage entre les candidats du PND eux-mêmes, plutôt qu'entre ces derniers et ceux des partis de l'opposition ${ }^{4}$.

Les principaux partis de l'opposition légale (Wafd, Tagammu' et parti nassérien) étaient en effet progressivement rentrés dans un rapport asymétrique avec le régime dont ils avaient accepté les règles du jeu clientéliste ${ }^{5}$. De l'ensemble de ces facteurs découlait le fait que ces partis disposaient non seulement de peu de ressources humaines, financières ou légales, mais qu'ils étaient en outre largement discrédités aux yeux d'une grande partie de la population, et en particuliers des opposants au régime. Enfin, la crise de la représentation politique induite par cette faiblesse des partis de l'opposition était encore aggravée par l'exclusion des islamistes hors du jeu politique légal.

Durant la première décennie du $\mathrm{XXI}^{\text {ème }}$ siècle, le discrédit qui frappe les partis d'opposition égyptiens s'étend progressivement à la forme partisane elle-même. Parmi les différentes organisations de l'opposition dépourvues de statut légal, seuls le Wasat et Karâma ${ }^{6}$ cherchent encore à se constituer en partis politiques légaux. Et encore les dirigeants de Karâma se satisfont-ils, à partir de 2004, de l'absence de reconnaissance légale, qu'ils utilisent comme argument de légitimation leur permettant de se présenter comme des adversaires résolus du régime, suffisamment représentatifs pour être considérés comme dangereux, et dès lors interdits.

Cette situation s'explique en partie par le fait que l'expérience militante de cette décennie a démontré l'efficacité - en termes de mobilisation - de formes alternatives d'organisation : comité populaire de soutien au peuple palestinien en 2001, Hamla Sha'abiya et Kefaya en 2004-2005, « mouvement du 6 avril » en 2008, Association nationale pour le changement en 2010. Toutes ces organisations, et de nombreuses autres moins connues, ont en commun le refus de se positionner sur le spectre idéologique, d'être créées autour d'un projet, d'être structurées en réseau, et enfin d'attirer - toujours davantage - de jeunes militants ${ }^{7}$. Durant la période 2009-2010, ces nouvelles organisations commencent visiblement à inquiéter les partis d'opposition les mieux établis (Wafd, Tagammu' et parti nassérien), et la presse se fait l'écho des débats qui opposent leurs dirigeants à des intellectuels engagés dans ces organisations ${ }^{8}$. Ces partis d'opposition ont néanmoins pris part même si avec un temps de retard - aux mobilisations de janvier-février $2011^{9}$.

4 Ben Nefissa Sarah et Alâ' Al-Din Arafat, Vote et démocratie dans l'Égypte contemporaine, IRD-Karthala, 2005.

5 Sur ce point, voir Kassem May, In the Guise of Democracy. Governance in Contemporary Egypt, Ithaca Press, 1999.

6 Ces deux partis ont été fondés au milieu des années 1990 par des militants dissidents des Frères musulmans (pour le Wasat) et du parti nassérien (pour Karâma). Les fondateurs de ces deux partis sont issus de la même génération militante, née du mouvement étudiant des années 1970. Ils reprochaient aux dirigeants plus âgés de leur organisations-mères respectives aussi bien leurs œillères idéologiques que leur incapacité à agir de concert avec les autres forces de l'opposition. La commission des partis a refusé à plusieurs reprises la création de ces deux partis, qui ne disposaient toujours pas, au moment où a éclaté la révolution, de statut légal.

7 Sur ce sujet voir (en arabe) l'ouvrage collectif dirigé par Dînâ Shahâta, Le retour de la politique. Les nouveaux mouvements contestataires en Égypte, Le Caire, Éditions du Centre d'études politiques et stratégiques de Al-Ahrâm, 2010.

8 Pour un article interrogeant la pertinence de la forme partisane, voir par exemple El-Shobaki Amr, "Parties, Movements, and Prospects for Change in Egypt" AlMasry AlYoum English Edition, 23 mai 2010. Pour un point de vue défendant les partis politiques et critiquant les nouveaux mouvements protestataires, voir l'interview accordée par le secrétaire général du Wafd Munîr Fakhrî 'Abd Al-Nûr à Al-Masrî Al-Yûm, publiée le 22 avril 2010.

9 Sur l'attitude des partis politiques égyptiens durant la révolution, voir les chapitres qui y sont consacrés (en arabe), 


\section{L'effervescence du printemps 2011}

Dans les mois qui suivent le départ de Moubarak, la forme partisane opère un retour en grâce spectaculaire auprès de l'ensemble des acteurs politiques, du fait des nouvelles conditions juridiques et politiques, éminemment plus favorables à la forme partisane, qui s'impose tout naturellement comme la forme privilégiée pour conquérir le pouvoir d'État, et ceci malgré la multiplication tout aussi spectaculaire des " coalitions de jeunes de la révolution », davantage perçues comme des instruments permettant de veiller sur le respect des principes de la révolution, au moyen de mobilisations ponctuelles dans les rues et sur les places du pays.

Dès le 19 février 2011, le Wasat est le premier parti à bénéficier des nouvelles conditions politiques, puisque le tribunal des partis du Conseil d'État lui accorde enfin son imprimatur en cassant la dernière décision de la commission des partis le concernant, concluant ainsi une bataille juridique longue de quinze années. Mais le véritable coup d'envoi à la création de nouvelles organisations partisanes est donné le le 28 mars 2011 par le Conseil supérieur des forces armées, qui promulgue à cette date une nouvelle loi des partis. Cette loi abolit en effet certains obstacles mis en place par la précédente législation (obligation d'originalité par exemple). Surtout, elle supprime le lien organique qui reliait le pouvoir exécutif à la commission des partis, désormais uniquement composée de juges indépendants. La nouvelle loi durcit l'ancienne législation sur un seul point : le nombre minimal de membres requis, qui passe de 2000 à 5000 . Mais il est sans doute plus facile de réunir 5000 membres pour un parti en avril 2011 que 2000 un an plus tôt.

Cette situation donne lieu à un véritable appel d'air, qui initie une quatrième vague de création de partis politiques en Égypte. Les deux premières vagues, dans les première années du $\mathrm{XX}^{\text {ème }}$ siècle puis dans l'entre-deux guerres, constituaient une réaction à la mainmise britannique sur les affaires de l'Égypte ${ }^{10}$. La troisième vague, à partir de 1978, avait au contraire été initiée par le haut, sous la pression de l'agenda néo-libéral. Il semble que la quatrième vague soit aujourd'hui le résultat d'une combinaison de ces deux facteurs: une pression internationale en faveur de la «bonne gouvernance » rencontrant l'aspiration de larges secteurs de la société à participer à la vie politique.

Ces conditions exceptionnelles expliquent sans doute le revirement de nombreux acteurs jusque là hostiles ou indifférents à la forme partisane : les Frères musulmans hésitaient depuis trente ans à créer un parti, du fait évidemment des contraintes autoritaires, mais aussi de la condamnation de la forme partisane par Hassan Al-Banna, le fondateur du mouvement, dont les textes sont toujours étudiés par les membres de la Confrérie dans le cadre des mécanismes de formation interne ${ }^{11}$. Les Frères sont cependant les premiers à bénéficier de la nouvelle loi des partis, avec l'autorisation qui leur est rapidement accordée de créer le parti de la Liberté et de la Justice (PLJ).

La création du parti de la Lumière (hizb al-nûr) intervient peu de temps après. Ce parti est basé sur l'organisation de prédication salafiste ( $a l-d a$ 'wa al-salafiya), dont l'attitude envers la politique avait auparavant toujours oscillé entre l'indifférence et la condamnation comme activité impie. De la même manière, alors que les Églises coptes avaient adopté depuis trois décennie une attitude extrêmement prudente à l'égard de la politique, la plus importante d'entre elle - l'Église orthodoxe se rallie presque ouvertement à l'initiative du milliardaire chrétien Naguib Sawiris, qui créé un parti politique libéral, le parti des Égyptiens libres. Quant aux «jeunes de la révolution», leur socialisation politique s'est faite bien souvent en opposition à la logique partisane, au sein des

dans Rabî' 'Amrû Hâshim (dir.), La révolution du 25 janvier. Une première lecture et une prospective, 3ème éd., Le Caire, Éditions du Centre d'études politiques et stratégiques de Al-Ahrâm, 2011.

10 Cf. notamment (en arabe) Shukr 'Abd Al-Ghafâr et al., Les partis politiques et la crise de la diversité en Égypte, Le Caire, Éditions du Centre de recherches arabes et africaines, 2010.

11 Sur les débats internes qui ont agité la Confrérie autour de l'opportunité de créer ou non un parti, voir par exemple (en arabe) Sâmî Ibrâhîm Al-Dalâl 'Abd Allah, Les islamistes et la démocratie en Égypte. Une tempête et un débris, Maktaba Madbûlî, 2007. Pour une analyse plus en profondeur des contradictions internes de la principale organisation islamiste égyptienne, voir (toujours en arabe) Tammam Hussam, L'évolution des Frères musulmans. La dislocation de l'idéologie et la fin de l'organisation, Le Caire, Maktaba Madbûlî, 2006. 
nouveaux mouvements contestataires évoqués plus haut. En 2011, cependant, les partis issus de cette mouvance se multiplient. Enfin, jusqu'en 2010, les soutiens du régime se satisfaisaient de la structure lâche que représentait le PND, sorte de fédération de réseaux clientélistes implantés localement. La dissolution de celui-ci le 16 avril pousse ses anciens membres à créer eux aussi leurs propres partis. À cela s'ajoutent enfin des initiatives sans lendemains électoraux, particulièrement à gauche, où des partis s'organisent autour de centres de recherche, de syndicats indépendants, de coopératives agricoles, etc.

\section{Les partis dans la bataille des législatives}

\section{Le rôle des partis dans la sélections des candidats et la mobilisation des électeurs}

Outre ce foisonnement de nouveaux partis, il faut compter avec les effets des nouvelles lois électorales, qui ont permis aux partis politiques de jouer au cours des élections législatives de 20112012 un rôle plus important que jamais.

La loi organisant les élections a favorisé les partis politiques, d'abord en prévoyant de réserver deux tiers des sièges au scrutin proportionnel. Si la possibilité existait de former des listes indépendantes, dans les faits la majorité des listes défendaient la couleur d'un parti (Wafd, Wasat, 'Ad1'2), même si ce parti pouvait en fait représenter une coalition (le PLJ et le parti de la Lumière représentaient respectivement «la coalition démocratique» et «l'alliance pour l'Égypte $»^{13}$ ). Inversement, l'étiquette d'une coalition pouvait en réalité désigner une liste mono-partisane. Ainsi, les partis du Bloc égyptien s'étaient répartis les différentes circonscriptions entre eux. De ce fait, leur liste pouvait être selon les circonscriptions une liste du parti des Égyptiens libres, une liste du parti égyptien social-démocrate, ou encore une liste du Tagammu'. La seule coalition à présenter ses listes comme telles était celle de "la révolution continue ${ }^{14}$. Les partis et les coalitions de partis disposent en effet d'une couverture médiatique nationale, ce qui les rendait plus intéressants pour les candidats potentiels que la possibilité de constituer des listes indépendantes. À défaut de décrocher l'investiture d'un parti, la meilleure stratégie alternative consistait ainsi à se rabattre sur une candidature individuelle.

Or, les circonscriptions individuelles se sont au final révélées immenses, deux fois plus grandes qu'initialement prévu dans l'avant-projet de loi de juin 2011, qui projetait de réserver deux tiers des sièges au scrutin individuel. Après trois mois de tractations avec les partis, les proportions se sont en effet inversées, et l'Égypte a été divisée en seulement 83 circonscriptions pour les sièges pourvus au scrutin individuel, chacune devant élire deux députés, dont l'un au moins devait appartenir à la catégorie " ouvriers et paysans ». De ce fait, ces circonscriptions sont devenues beaucoup moins abordables pour les petits notables locaux, dont les ressources et la notoriété ne leur permettaient pas d'espérer conquérir une circonscription de près d'un million d'habitants, d'autant que l'expérience du référendum de mars 2011 laissait présager un taux de participation bien supérieur à celui que l'on pouvait enregistrer avant la révolution.

Les partis ont ainsi joué un rôle crucial dans ces élections, et les candidats potentiels se sont vus

12 «Parti de la Justice». Fondé par des proches de l'ancien directeur de l'Agence internationale de l'énergie atomique et prix Nobel de la Paix Muhammad Al-Baradei, ce parti libéral centriste s'est par la suite éloigné de ce dernier et a présenté dans tous le pays des candidats issus de la jeunesse révolutionnaire.

13 "La coalition démocratique » avait au départ vocation a devenir un rassemblement des principales forces politiques du pays. Le retrait du Wafd, puis des salafistes et du Wasat, en ont fait une alliance électorale dominée par les Frères. Outre ces derniers, ses principaux membres étaient Karâma et le parti du Travail, parti islamo-nationaliste et socialiste, résurgence de l'organisation de l'entre-deux guerre Jeune Égypte. Si le Wafd et le Wasat ont décidé de concourir seuls - devenant respectivement la troisième et la cinquième forces représentées au Parlement - les salafistes (parti de la Lumière et parti de la Vertu) se sont rassemblés au sein de « l'alliance pour l'Égypte », à laquelle se sont joints les anciens jihadistes des Gama 'ât Islamiya, réunis au sein du parti de la Construction et du Développement.

14 Rassemblant cinq partis socialistes ou libéraux, ainsi que « la coalition des jeunes du 25 janvier ». 
contraints de rechercher leur investiture. Ces élections ont ainsi constitué le cadre d'un sorte de recyclage des notabilités, sous la bannière de divers partis. Les notables locaux avaient en effet le choix entre deux types de stratégies. Pour les petits notables, les deux alternatives étaient soit de faire campagne seuls, en indépendants, sans aucune chance de succès, dans une stratégie d'augmentation de leur visibilité sociale, en vue notamment d'échéances futures, soit de concourir sur la liste d'un parti, plusieurs d'entre eux cherchant à recruter des notables dans différentes quartiers de chaque circonscription, dans le but d'étendre leur ancrage local. Pour les notables disposant de davantage de moyens (hommes d'affaires, chefs de tribus), le choix consistait à se présenter seuls, de préférence avec le soutien d'un parti afin de légitimer leur candidature, ou à prendre la tête d'une liste, afin de maximiser leurs chances d'êtres élus, et éventuellement de servir de locomotive à leurs listes. Enfin, les candidats les plus compromis avec l'ancien régime devaient se résigner à se présenter seuls, ou à rechercher le soutien d'un des partis issus de l'ancien PND.

L'investiture accordée par les partis permettait en effet de bénéficier des retombées des campagnes médiatiques menées au niveau national, de donner un verni de légitimité idéologique à des notables jusqu'alors étrangers à ce type de considérations, et de profiter des capacités de mobilisation de ces partis. Outre leur rôle dans la campagne électorale, ceux-ci sont en effet parfois allés jusqu'à mobiliser physiquement les électeurs, en affrétant des microbus pour les conduire jusqu'aux bureaux de vote. De fait, les candidats indépendants ont été balayés au cours de ces élections : 23 députés indépendants sur 498, alors que lors des précédentes élections, la majorité des députés étaient des indépendants, qui ne se ralliaient au PND qu'une fois élus ${ }^{15}$.

\section{La relative autonomie des organisations partisanes}

Cependant, les partis politiques égyptiens ne sont encore que très faiblement autonomisés par rapport à leurs milieux partisans respectifs. Le PLJ, pour commencer, a été créé par décision du conseil consultatif (majlis al-shûrâ) des Frères musulmans, qui a également élu ses dirigeants et choisi son candidat à l'élection présidentielle. Pendant la campagne, et jusqu'au jour même du vote, des voitures surmontées d'un mégaphone circulaient dans les rues pour rappeler que le PLJ est le parti des Frères musulmans. Pour contourner la loi interdisant le recours à des slogans religieux, la Confrérie imprimait des affiches aux couleurs du parti, mais sans faire explicitement mention de celui-ci, qui proclamaient que "l'islam est la solution». Dans les mosquées, les imams de la Prédication salafiste appelaient à voter pour le parti de la Lumière, et à se joindre à ses manifestations. Dans les quartiers majoritairement chrétiens, l'Église donnait des instructions aux cadres du parti des Égyptiens libres, notamment concernant le choix des candidats à soutenir au second tour. Les anciens du PND, de leur côté, n'hésitaient pas à mobiliser leurs 'asabiya ou à faire voter leurs employés, recourant eux aussi - lorsqu'ils en avaient les moyens - aux microbus pour convoyer les électeurs jusqu'aux bureaux de vote. Enfin, concernant les jeunes de la révolution, si «le mouvement du 6 avril» ne donnait officiellement pas de consignes de vote, ses membres participaient souvent à la campagne à titre individuel, la plupart du temps pour les candidats de « la révolution continue » lorsqu'il y en avait. Ces derniers pouvaient d'ailleurs être des membres du « 6 avril » ou d'autres coalitions de jeunes, s'étant retirés de leur mouvement le temps de la campagne.

Ainsi, si les circonstances historiques et les règles du jeu politique ont rendu les partis incontournable lors de ces élections, elles n'ont néanmoins pas fait d'eux les seuls maîtres du scrutin, et les cadres et militants des partis ont dû composer avec différentes puissances sociales porteuses de logiques concurrentes. Si les élections constituent habituellement un catalyseur de la construction et de l'implantation locale des organisation partisanes ${ }^{16}$, il est à noter que dans le cas de l'Égypte, ce processus prend appui sur des logiques et des organisations pré-existantes extrêmement variées: tribalisme, clientélisme, affairisme, charité, prédication religieuse, militantisme

15 Ben Nefissa Sarah et Alâ' Al-Din Arafat, op. cit.

16 Rokkan Stein et Seymour M. Lipset. Structures de clivages, systèmes de partis et alignement des électeurs : une introduction. Éditions de l'université de Bruxelles, 2008. 
révolutionnaire, etc ${ }^{17}$. Dans ces conditions, les partis qui ont le mieux réussi sont ceux qui étaient capables de présenter des notables qui ne soient pas des ralliés de dernière minute : la puissance socio-économique des Frères musulmans, ainsi que la force de frappe religieuse des salafistes et leurs liens avec l'administration, en ont ainsi fait les nouveaux partis dominants.

Le Wafd, ce vieux parti de la bourgeoisie terrienne implanté à l'échelon local a pu de son côté obtenir une troisième place grâce à son maillage territorial et à la réputation bien ancrée de son nom et de son image. Le succès le plus intéressant, en terme de sociologie électorale, reste cependant celui du Bloc égyptien, adossé au parti des Égyptiens libres. Fondé par un milliardaire chrétien, ce parti a su attirer une partie de la bourgeoisie d'affaires, de ses cadres et de ses philanthropes. L'expérience entrepreneuriale et/ou caritative de ses cadres, couplée avec le soutien officieux de l'Église, lui a permis de créer rapidement une armature organisationnelle présente dans l'ensemble du pays. Le parti a su ensuite recruter des petits notables locaux en provenance de différents quartiers, afin non seulement de profiter de leur potentiel de mobilisation électorale, mais aussi de s'implanter dans ces quartiers et d'y faire connaître rapidement le nom, le programme et les symboles du parti. Cette stratégie d'instrumentalisation des logiques clientélistes au profit d'une logique partisane a permis à ce parti de se structurer et de s'implanter en un temps record, et laisse espérer à ses cadres qu'ils seront en mesure de se passer de l'appui des notables lors des prochaines échéances électorales, et d'accéder ainsi eux-mêmes à des charges électives.

17 Pour un aperçu de ce phénomène à l'échelle du monde arabe, consulter l'ouvrage collectif dirigé par Myriam Catusse et Karam Karam, Returning to Political Parties?, Lebanese Center for Policy Studies, 2010. 